\title{
Variability Studies and Asteroseismology with the XMM Optical Monitor
}

\section{S. Horner}

\author{
Department of Astronomy and Astrophysics, Penn State University, \\ University Park, PA 16802, USA
}

E. Antonello

\author{
Osservatorio Astronomico di Brera, Milano-Merate, Italy
}

\begin{abstract}
It will be possible to make photometric variability studies from space of stars serendipitously falling in the field of view of the Optical Monitor, and to search for asteroseismological phenomena in bright target stars.
\end{abstract}

\section{Optical Monitor}

The Optical Monitor (OM) (cf. Horner et al. 1994) is one of three instruments on ESA's XMM observatory (cf. Mason et al. 1995) to be launched in 1999. The $\mathrm{OM}$ is a $30 \mathrm{~cm}$ aperture Ritchey-Chrétien telescope for UV and optical band observations that is co-aligned with the X-ray telescopes. It has two detectors for photometric and low resolution spectroscopic observations. A beam splitter separates the light into blue $(160-550 \mathrm{~nm})$ and a red beams $(500-1100 \mathrm{~nm})-$ the blue detector is a Micro-channel plate Intensified CCD (MIC), while the red detector is a frame transfer CCD.

The fields of view of the two detectors are similar, approximately 17 arcmin square. The blue detector (MIC) has a resolution of 1 arcsec, while the red detector has a resolution of 3.4 arcsec. The 11 position filter wheels in each of the beams carry broad and medium band filters, grisms and a magnifier; the red beam filter wheel also carries a defocusing lens.

It will be possible to perform variability studies from space of stars serendipitously falling in the field of view of the Optical Monitor, and to search for asteroseismological phenomena during long observations by XMM to detect the coronal X-ray spectra of bright stars. Monte Carlo simulations using the Guide Star Catalog (Jenkner et al. 1990 \& Lasker et al. 1988) predict that if XMM's pointings are randomly distributed on the sky, an average 0.85 stars of $m_{V} \leq$ 12 will fall into the field of view per pointing. If the pointings are randomly distributed within $15^{\circ}$ of the galactic plane, an average 1.3 stars of $m_{V} \leq 12$ will fall into the field of view per pointing. 


\section{Photometric precision}

The expected differential photometry precision and minimum detectable amplitude for bright stars observed with the red detector are reported in Table 1. The $1 \sigma$-precision has been estimated for a source temperature of $5770 \mathrm{~K}$. Owing to the brightness of the stars, the single exposures are assumed very short, $1 \mathrm{~s}$ or less, and the star image is spread over many pixels. The estimates have been made for a cumulative number of exposures of $30 \mathrm{~s}$. In general, the effects of readout noise due to the very high cumulative number of exposures are not significant for very bright stars. The precisions have been estimated assuming a perfect stabilization of the images and of CCD temperature. Moreover, no contamination owing to cosmic rays and no degrading of the CCD performances due to radiation have been taken into account. It will be possible to correct for the cosmic ray contamination by means an on board frame comparison technique only for images of faint sources with relatively long exposure times; it will not be possible to apply a similar correction to images with short exposure times. Therefore, we expect an incidence of about a few percent of the time series data to be contaminated by cosmic rays in the case of bright star observations.

Table 1. Differential photometry precision and minimum detectable amplitude (see text)

\begin{tabular}{lcc}
$\mathrm{m}_{V}$ & precision $(1 \sigma)$ & amplitude \\
\hline 4 & $9.3 \mathrm{E}-5$ & $8.9 \mathrm{E}-6$ \\
5 & $1.5 \mathrm{E}-4$ & $1.5 \mathrm{E}-5$ \\
6 & $2.3 \mathrm{E}-4$ & $2.2 \mathrm{E}-5$ \\
7 & $3.7 \mathrm{E}-4$ & $3.5 \mathrm{E}-5$ \\
8 & $5.9 \mathrm{E}-4$ & $5.6 \mathrm{E}-5$ \\
9 & $9.3 \mathrm{E}-4$ & $8.9 \mathrm{E}-5$ \\
10 & $1.5 \mathrm{E}-3$ & $1.5 \mathrm{E}-5$ \\
11 & $2.3 \mathrm{E}-3$ & $2.2 \mathrm{E}-4$
\end{tabular}

Acknowledgments. Work in the US is supported by NASA contract NAS531787 at Penn State. In Italy, financial support is provided by ASI, in the UK by SERC, and in Belgium by the Belgian Ministry of Science.

\section{References}

Antonello, E., \& Cropper, M.S. 1992, in Stellar Photometry - Current Techniques and Future Developments, Butler, C.J., Elliott, I., Cambridge Univ. Press, 358

Horner, S.D., et al. 1994, in Instrumentation in Astronomy VIII, Crawford, D.L., Craine, E.R., SPIE, 2198, 1238

Jenkner, H., et al. 1990, ApJ, 99, 2081

Lasker, B.M., et al. 1988, ApJS, 68, 1

Mason, K.O., et al. 1995, Adv. Space Res., 16, 41 